\section{Auswirkungen eines verstärk- ten Anbaus nachwachsender Rohstoffe im globalen Maßstab}

von Stefan Bringezu und Helmut Schütz, Wuppertal Institut für Klima, Umwelt, Energie

\begin{abstract}
Weltweit wächst der Druck zur Ausweitung landwirtschaftlicher Flächen auf Kosten natürlicher Ökosysteme vor allem durch die steigende Nachfrage nach tierbasierter Nahrung und künftig verstärkt durch den Anbau von Energiepflanzen. Zusätzliche Treibhausgas-Emissionen und andere Umweltbelastungen sind die Folge. Die (Flächen-) Konkurrenz zwischen Nahrungsmittelverfbrauch einerseits und zwischen energetischem und stofflichem Einsatz von Non-Food-Biomasse andererseits wächst. Produktionsseitige Strategien haben eine begrenzte Wirkung auf die globale Flächennutzung. Vielmehr ist ein ressourceneffizienterer Verbrauch notwendige Voraussetzung für eine nachhaltige Biomassenutzung. Der Beitrag erläutert dies an Hand umfassender Szenarien und gibt konkrete Politikempfehlungen.
\end{abstract}

\section{Einleitung}

Die Europäische Union und die deutsche Bundesregierung streben einen erheblichen Ausbau der energetischen Nutzung von Biomasse vor allem im Kraftstoffbereich an. Auch in den USA, in China, Indien, Brasilien und anderen Weltregionen werden zunehmend nationale Ziele zur verstärkten Nutzung von Biomasse für energetische Zwecke verfolgt. Dabei liegt der Schwerpunkt auf Biokraftstoffen, wobei sich abzeichnet, dass Länder mit hohem Biomassepotenzial (wie z. B. in den Tropen) nicht nur die eigene Versorgung zum Ziel haben, sondern verstärkt die Importbedarfe der Industrieländer bedienen möchten. Die steigende Nachfrage nach Biokraftstoffen der ersten Generation verschärft damit die wachsende Nutzungskonkurrenz um Anbauflächen zur Ernährung einerseits und Naturflächen andererseits.
Eine Nutzungskonkurrenz ergibt sich vor allem bei land- und forstwirtschaftlich angebauten Rohstoffen, die für verschiedene Verarbeitungspfade genutzt werden können. So kann Weizen als Rohstoff für die Produktion von Mehl und daraus erzeugten Lebensmitteln dienen. Er dient jedoch auch als Kraftfutter. Zudem wird er eingesetzt zur Stärkeherstellung. Diese Stärke kann wiederum als Lebensmittelzusatz dienen oder für die Herstellung von Papier und Pappe. Zudem wird Weizen auch zur Bioethanolproduktion eingesetzt.

Die Konkurrenz zwischen den verschiedenen Nutzungen kann direkt oder indirekt wirken. Eine direkte Konkurrenz liegt vor, wenn Rohstoffe oder Grundstoffe für mehr als einen Nutzungspfad eingesetzt werden können beispielsweise wenn Mais als Futtermittel dient, zur Biogasproduktion angebaut wird oder als Grundstoff für die Stärkeherstellung in der chemischen Industrie dient.

Eine indirekte Konkurrenz liegt vor, wenn die verschiedenen Roh- oder Grundstoffe auf gleiche Flächenressourcen angewiesen sind. Das gilt für alle ackerbaulich produzierten Rohstoffe inkl. der daraus hergestellten Grundstoffe und generell für all jene Rohstoffe, die das primäre Ziel einer land- oder forstwirtschaftlichen Flächenbewirtschaftung darstellen. Das bedeutet, dass auch die Produktion von Energiepflanzen, die zunächst nicht für Nahrungsmittelzwecke und nicht für industriell-stoffliche Verwendungen angebaut werden, doch mit diesen Aktivitäten in Konkurrenz stehen, da sie auf die gleichen Anbauflächen zurück greifen. Die Flächenkonkurrenz kann sich dabei zum einen im Inland und zum anderen im Ausland auswirken. Kurz- bis mittelfristig führt der verstärkte Zugriff auf nachwachsende Rohstoffe über die Flächenkonkurrenz zunächst zu steigenden Preisen aller vorwiegend ackerbaulich produzierten Rohstoffe und bewirkt so auch eine Verschiebung der Kostenstruktur in den Industriezweigen, die wesentlich von der Verarbeitung dieser Rohstoffe und davon abgeleiteter Grundstoffe abhängen. Langfristig führt eine zunehmende Flächenkonkurrenz zu einer Verschiebung der Flächennutzungstypen, im konkreten Fall der Energiepflanzen zu einer Ausdehnung der globalen Ackerfläche zu Lasten von natürlichen Ökosystemen. 


\section{Ernährung und weltweite Verfügbarkeit landwirtschaftlicher Flächen}

Ein zentrales Kriterium für die Bewertung globaler Ressourcennutzung ist die heute und in Zukunft pro Kopf der Weltbevölkerung zur Verfügung stehende landwirtschaftlich intensiv bewirtschaftete Nutzfläche; diese wird hier definiert als „Anbauland“, das Ackerland und Dauerkulturen einschließt. Diese lag 2004 bei etwa $2.500 \mathrm{~m}^{2}$ pro Person. Nach Angaben der UN und Prognosen der Welternährungsorganisation (FAO) wird die Weltbevölkerung bis 2030 gegenüber 2004 um etwa 30 Prozent anwachsen und die Hektarerträge für Getreide werden um ca. 29 Prozent steigen (FAO 2003, FAO 2006), d. h. in etwa werden diese beiden Entwicklungen miteinander Schritt halten (siehe Abb. 1). Daten der FAO zeigen auch, dass im Zeitraum 1961 bis 2006 die Getreideerträge zwar gestiegen sind, diese Zunahme sich aber in der letzten Dekade abgeschwächt hat (Hazell, Wood 2008), so dass eine Fortschreibung linearer Steigerungsraten oder Annahmen sogar höherer Steigerungen der durchschnittlichen Erträge als wenig realistisch und unsicher eingestuft werden müssen. 2030 werden pro Person der Weltbevölkerung nur noch etwa 2.000 $\mathrm{m}^{2}$ intensiv bewirtschaftete Landwirtschaftsfläche zur Verfügung stehen, selbst wenn man nach FAO-Prognosen eine absolute Ausdehnung der Anbaufläche um 120 Mio. ha annimmt. Damit wäre pro Kopf ein Fünftel weniger nutzbar als im Jahr 2004.

Der Fleischkonsum in Entwicklungsländern wird im selben Zeitraum um 55 Prozent steigen, während der Getreidekonsum „,nur“ um ca. 28 Prozent zunehmen wird. Da die Tierproduktion für die Erzeugung der Futtermittel erheblich mehr Fläche beansprucht als der Anbau für die direkte pflanzliche Ernährung des Menschen, wird sich allein durch diese Entwicklung der Druck zur Ausweitung der landwirtschaftlichen Flächen auf Kosten natürlicher Ökosysteme deutlich erhöhen. Das Ausmaß der Flächenmehrbeanspruchung für höheren Fleischkonsum gegenüber pflanzlicher Ernährung hängt zum einen vom Mix der konsumierten Fleischsorten ab, zum anderen von Faktoren wie den Erträgen verschiedener Futtermittel und der Effizienz der Futterverwertung. Zur ersten Orientierung können Angaben für Deutschland dienen, wonach der Konsum tierisch basierter Nahrungsmittel pro Nährwert (in cal) einen 4,8-fach höheren Flächenbedarf aufweist als der Konsum pflanzlicher Nahrungsmittel (basierend auf Busch 2008). Es wird darüber hinaus erwartet, dass auch in den so genannten Hungerländern der Nahrungsmittelverbrauch in Zukunft deutlich ansteigen wird und damit tendenziell weiterer

\section{Abb. 1: Globale Entwicklung der Bevölkerung, der Getreideerträge, der Anbauflächen* und des Fleischkonsums in Entwicklungsländern bis 2030}

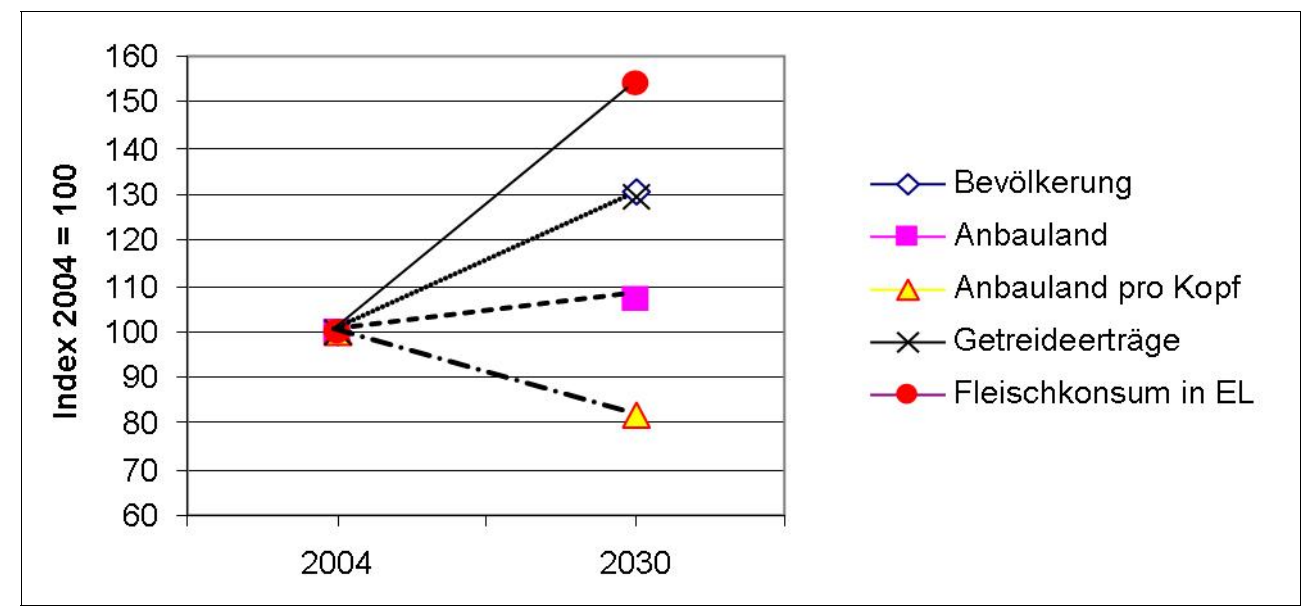

* Ackerland und Dauerkulturen

Quellen: UN-Bevölkerungsstatistik; FAO 2003; FAO 2006 
zusätzlicher Anbauflächenbedarf (für Ackerland und Dauerkulturen) entsteht, ohne dass dieser derzeit quantitativ eingeschätzt werden könnte (OECD 2008).

Dies bedeutet, dass allein aufgrund der Nachfrageentwicklung für die Ernährung ein wachsender Druck in Richtung weiterer Ausweitung der Anbauflächen entstehen wird. Die Nachfrage nach Biokraftstoffen auf der Basis von Energiepflanzen erhöht den Druck auf die knapper werdende landwirtschaftlich nutzbare Fläche zusätzlich. Faktoren wie die Zunahme der Bodendegradation oder Auswirkungen des Klimawandels stellen in diesem Zusammenhang ein weiteres schwer kalkulierbares Risiko für die Flächennutzbarkeit und die Ertragssteigerungen dar.

\section{Konkurrenz zwischen stofflicher und energetischer Nutzung nachwachsender Rohstoffe}

Nachwachsende Rohstoffe nehmen einen zunehmenden Anteil der Anbaufläche in Deutschland in Anspruch. Im Jahr 2006 wurden bereits 1,6 Mio. ha hierfür belegt; dies waren ca. 13 Prozent des gesamten Ackerlandes, wobei wiederum mehr als 80 Prozent für die energetische Nutzung bestimmt waren. Es wird erwartet, dass die weitere Entwicklung vor allem eine Ausdehnung des Energiemaisanbaus von derzeit rd. 250.000 auf annähernd eine Million Hektar zur Folge hätte (Wuppertal Institut et al. 2008): Dies wird u. a. auf Kosten der Anbaufläche für Getreide geschehen (Gömann et al. 2007). Mit der Ausdehnung des Energiepflanzenanbaus wären auch erhebliche indirekte Nutzungskonkurrenzen um Anbaufläche zu erwarten, die zur Verschärfung der Konkurrenz zwischen verschiedenen Verwendungen der landwirtschaftlichen Produktion beitragen würden.

Die Konkurrenz zwischen primär stofflichem und energetischem Einsatz von Biomasse untersuchten Wuppertal Institut und RheinischWestfälisches Institut für Wirtschaftsforschung (Essen) (Wuppertal Institut, RWI 2008). Für die landwirtschaftlichen Rohstoffe ergaben sich für Deutschland folgende Trendaussagen:
1. Weizen: Die stoffliche Verwendung wird zunehmend mit direkten energetischen Nutzungskonkurrenzen, vor allem zur Bioethanol- und Wärmeproduktion konfrontiert werden. Sollten zudem die sehr hohen Potenziale zur Verwendung von Weizenstärke für Biopolymere tatsächlich erreicht werden (Meo et al. 2007), so würden sich die direkten Nutzungskonkurrenzen erheblich verschärfen. Bei gleich bleibendem Bedarf an Weizen für Futter und Nahrung und nicht proportional wachsenden Hektarerträgen, würde die gesamte Versorgung in diesem Extremfall stark von Importen abhängig werden.

2. Mais: Vor allem indirekte Nutzungskonkurrenzen auf energetischer Ebene könnten die zukünftige stoffliche Verwendung von Körnermais weiter einschränken, sofern die sich abzeichnende Ausdehnung der Anbauflächen für Energiepflanzen (z. B. gefördert durch das EEG) verstärkt auf Kosten der Anbaufläche von Körnermais für die stoffliche Verwendung zur Herstellung technischer Stärke gehen sollte. Dies dürfte auch den Einsatz von Körnermais als Futtermittel und für Nahrungszwecke betreffen, so dass sich mögliche Preiseffekte und eine Verschiebung Richtung Import ergeben könnten.

3. Rohstoffe für Bioethanol: Bioethanol lässt sich weitaus kostengünstiger (etwa um den Faktor drei) in Brasilien erzeugen und importieren, als es mit heimischen Rohstoffen im Inland der Fall ist. Es ist zu erwarten, dass ab 2020 verstärkt Bioethanol aus $\mathrm{Zu}$ ckerrohr direkt importiert wird - vorausgesetzt die Handelsbarrieren werden abgebaut. Die Rohstoffe Kartoffeln sowie Zuckerrüben und Zuckerrohr sind für die energetische Nutzung und Konkurrenzbeziehungen innerhalb von Deutschland zu vernachlässigen.

4. Raps: Perspektivisch ist anzunehmen, dass sich die Produktion von alternativen Kraftstoffen auch weiterhin stärker an Dieselsubstituten orientieren wird. Rapsöl aus deutschem Anbau wird unter den gegebenen Rahmenbedingungen auch zukünftig überwiegend energetisch genutzt werden, die Entwicklungspotenziale der stofflichen Nutzungen bleiben dahinter deutlich zurück. Es ist zu erwarten, dass diese direkten Nutzungskonkurrenzen sich verstärkt auf die Kosten von Raps zur Nahrungsmittelproduk- 
tion auswirken werden. Zudem muss ab 2010 bereits mit verschärften indirekten Nutzungskonkurrenzen gerechnet werden, da dann aller Voraussicht nach die Anbauflächen für Raps in Deutschland infolge Fruchtfolgeeinschränkungen nicht mehr weiter ausgedehnt werden können. Die maximal mögliche Anbaufläche wird auf 1,6 bis 1,8 Mio. ha geschätzt, in 2008 wurden jedoch bereits 1,4 Mio. ha Raps angebaut.

5. Palmöl: Die Umsetzung der geplanten Ausweitung des Einsatzes von Palmöl in Blockheizkraftwerken kann derzeit nicht konkret eingeschätzt werden und hängt vor allem von laufenden Überlegungen zu politischen Regelungen im Rahmen internationaler Abkommen, eines novellierten EEG und der Wirksamkeit von Standards im Rahmen der geplanten Nachhaltigkeitsverordnung ab. Gegenüber einheimischem Rapsöl stellt Palmöl eine direkte Konkurrenz dar, die in den BAU-Szenarien (Wuppertal Institut et al. 2008) sogar deutlich dominieren würde. ${ }^{1}$ Inwiefern sich durch die zunehmende Produktion von Biodiesel aus Palmöl Nutzungskonkurrenzen zur klassischen stofflichen Verwendung von Palmöl in der chemischen Industrie ergeben, bleibt $\mathrm{zu}$ untersuchen. Bislang beruht der Zuwachs eher auf der Ausweitung von Anbauflächen.

6. Soja: Es wird erwartet, dass zukünftig insbesondere Brasilien und Argentinien auch für den Export große Mengen Biodiesel aus Soja herstellen werden. Bereits heute trägt Biodiesel aus Soja signifikant zum Biodieselabsatz in Deutschland bei. Bei Soja sind insbesondere Konkurrenzeffekte im Futter- bzw. Nahrungsmittelbereich zu erwarten. Da der Kraftstoffertrag pro Fläche bei Soja sehr gering ist (nur ein Achtel des Ertrags von Palmöl) sind die Flächenerfordernisse enorm.

\section{Flächenbedarf für nachwachsende Rohstoffe}

Zum Flächenbedarf der aktuellen und künftig $\mathrm{zu}$ erwartenden weltweiten Nachfrage nach nachwachsenden Rohstoffen liegen bislang keine belastbaren Abschätzungen vor. Für die EU und Deutschland können dagegen Aussagen getroffen werden. Für die EU-25 wurde die Verwendung von Biokraftstoffen 2020 mithilfe des „European Simulation Model“ untersucht (EC 2006). Die Ergebnisse zeigen, dass ein höherer Anteil von Biokraftstoffen am Kraftstoffverbrauch für Transporte bis 2020 von 7 bzw. 14 Prozent einen höheren globalen Flächenbedarf der EU-25 zur Folge hätte. Bei einem Biokraftstoffanteil von 7 Prozent läge der Flächenbedarf bei 14,8 Mio. ha und bei einem Anteil von 14 Prozent in Abhängigkeit vom Anteil importierter Biokraftstoffe zwischen 23,1 und 27,6 Mio. ha. Ein bis zwei Drittel dieser Flächeninanspruchnahme würden im Ausland erfolgen.

Für Deutschland wurden vom Wuppertal Institut zusammen mit UMSICHT und IFEU Szenarien über die globale Flächeninanspruchnahme zur Deckung des deutschen Biomassebedarfs für Ernährung und NichtErnährungszwecke hergeleitet (Wuppertal Institut et al. 2008). Dabei wurden zwei Business-as-usual-Szenarien (BAU I und BAU II) mit unterschiedlichen Annahmen zur Entwicklung der Nutzung nachwachsender Rohstoffe sowie zur Umsetzung der rechtlichen Regelungen und politischen Zielvorgaben zum Einsatz von Biomasse analysiert. Die beiden Szenarien unterscheiden sich im Hinblick auf den jeweils unterstellten Anteil an nachwachsenden Rohstoffen. Die Ergebnisse zeigen, dass ein erhöhter Anteil an Biokraftstoffen auch für Deutschland zu einer erheblichen Ausweitung des globalen „Flächenrucksacks“ führen würde, da der Biokraftstoffbedarf 2030 nur zu etwa einem Fünftel durch inländische Produktion gedeckt werden könnte. Der Import von Biokraftstoffen hätte zur Folge, dass die Flächenbelegung für den Konsum aller agrarischen Waren von rund $2.500 \mathrm{~m}^{2} \mathrm{im} \mathrm{Jahr}$ 2004 - dies entspricht in etwa der heute global pro Kopf verfügbaren Anbaufläche - auf 2.800 bis $2.900 \mathrm{~m}^{2}$ pro Person (BAU I bis II) im Jahr 2030 steigen würde (siehe Abb. 2 nächste Seite). Damit würde der Flächenbedarf die im Inland verfügbare Anbaufläche netto um etwa ein Drittel übersteigen (30 bis 36 Prozent; BAU I bis II)

Diese höhere globale Flächeninanspruchnahme von Deutschland im Jahr 2030 würde die dann weltweit verfügbare intensiv genutzte Anbaufläche (v. a. Ackerfläche) von ca. $2.000 \mathrm{~m}^{2}$ pro Person deutlich übersteigen. 
Abb. 2: Entwicklung des globalen Flächenbedarfs zur Deckung des deutschen Bedarfs an Biomasse für Ernährung, Energie und stoffliche Verwendungen

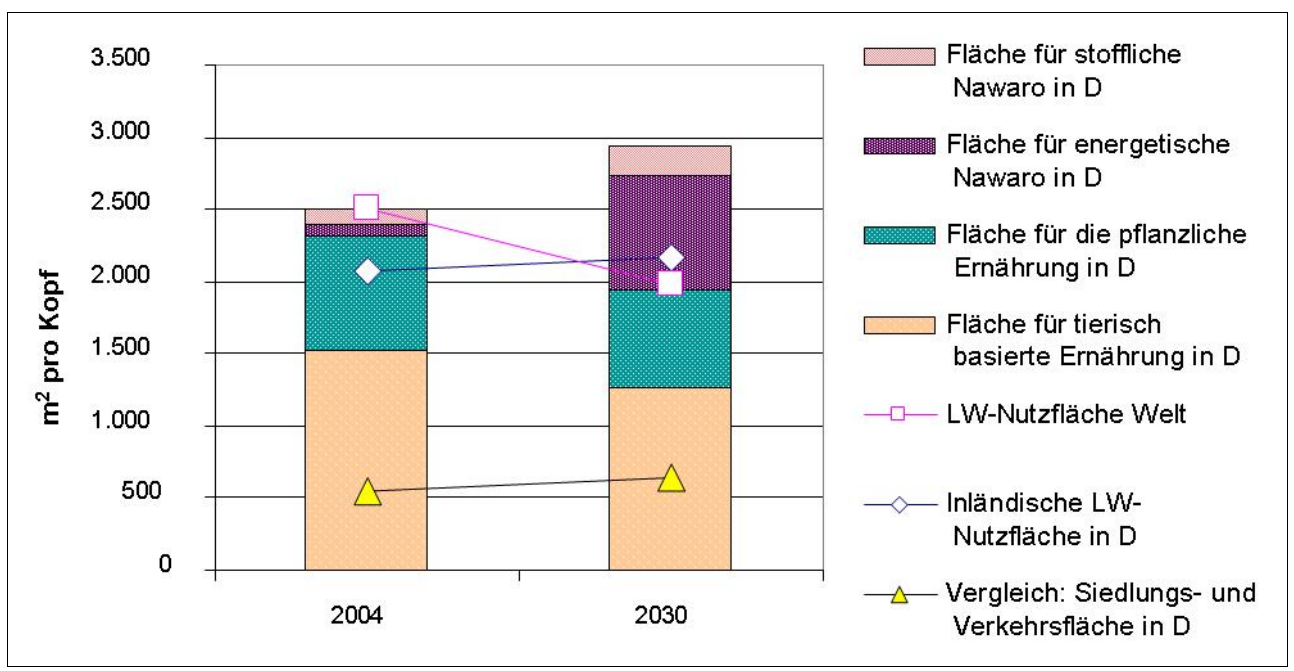

Quelle: Eigene Darstellung

Deutschland würde also bei fortgesetzt steigender Nachfrage nach Biokraftstoffen den Druck zur Ausweitung der globalen Anbaufläche deutlich erhöhen.

\section{Auswirkungen auf Agrarpreise und landwirtschaftliche Einkommen}

Nach Einschätzungen von Wuppertal Institut und RWI Essen (2008) werden die verschiedenen Ausbauziele für Bioenergie zu Preissteigerungen für Agrarprodukte führen. Dies gilt nicht nur für nachwachsende Rohstoffe, sondern auch prinzipiell für alle anderen Agrarrohstoffe. Befördert wird diese Entwicklung durch die ökonomischen Anreize zum Anbau von Energiepflanzen sowie Vorgaben durch nationale Bioenergieziele und z. B. Biokraftstoffquoten in Ländern wie USA oder Deutschland. Eine Verlagerung der Rohund / oder Kraftstoffversorgung in ökonomisch günstiger produzierende Entwicklungsländer wäre eine mögliche Konsequenz, um Preissteigerungen zu begegnen, was allerdings wiederum verbunden wäre mit gravierenden Umwelt- und Sozialrisiken.

$\mathrm{Zu}$ ähnlichen Einschätzungen kommen auch Studien von FAO / OECD sowie das International Food Policy Institute (OECD-FAO
2007; Rosegrant et al. 2006). ${ }^{2}$ Den kritischen Stimmen wird häufig entgegen gehalten, dass der Anbau von Energiepflanzen die Einkommenschancen der Landwirtschaft erhöht und damit die ländliche Entwicklung fördern kann (UN-Energy 2007). Ein solcher Effekt ist für Länder wie Deutschland durchaus zu erwarten (Wuppertal Institut und RWI 2008), doch ist zugleich zu bedenken, dass die durch direkte und indirekte Subventionen der Bioenergie erhöhten Preise bei gegebenem Volkseinkommen zu verminderten Ausgaben der Haushalte für nicht-energetische Produkte führen und sich damit negative Auswirkungen in anderen Branchen ergeben (Nusser et al. 2007).

In Entwicklungsländern sind oft sehr unterschiedliche Bedingungen gegeben. Häufig trägt ein erweiterter Energiepflanzenanbau, der sich ökonomisch rechnet, nach den bisherigen Erfahrungen eher zur Förderung weniger Großbetriebe bei. Kleinbauern werden eher verdrängt und müssen zudem höhere Preise für Nahrungsmittel zahlen (Biofuelwatch et al. 2007). Positive Beispiele wie das Sozialsiegel in Brasilien, bei dem Kleinbauern der Absatz von Pflanzenöl für Treibstoffe garantiert wird, sind von staatlicher Intervention abhängig und bislang die große Ausnahme. 


\section{Auswirkungen auf Naturschutz, Klimaschutz und Wasserressourcen}

Szenarien der UN (Millenium Ecosystem Assessment), von UNEP (Global Environment Outlook - GEO4) sowie anderer global ausgerichteter Studien und Untersuchungen verschiedener Autoren wie z. B. Searchinger et al. (2008) illustrieren, dass der zunehmende Flächenbedarf für nachwachsende Rohstoffe und Nahrungsmittel tierischer Herkunft zur globalen Ausweitung der landwirtschaftlichen Nutzfläche führen kann. Die Ausdehnung der Anbauflächen von Soja, Zuckerrohr und Palmöl dürfte vorwiegend auf Kosten von Wäldern, Savannen und Grasland insbesondere in Südamerika (v. a. Brasilien), Indonesien und Malaysia erfolgen und in Konkurrenz mit Naturschutzerfordernissen treten (UN-Konvention zum Schutz der Biodiversität). Aktuelle Entwicklungen bestätigen diese Annahmen: Die Erweiterung des Palmölanbaus in Indonesien beruht derzeit zu zwei Dritteln auf der Zerstörung von Regenwald, die restliche Anbauflächenausweitung von einem Drittel findet auf bereits vorher genutzten oder brachliegenden Flächen statt (Grieg-Gran et al. 2007). Die geplante Erweiterung des Anbaus von Ölpalmen in Indonesien um weitere 20 Mio. ha gegenüber dem Bestand von mindestens 6 Mio. ha (Colchester et al. 2006) dürfte ebenfalls zu großen Teilen zu Lasten von Naturflächen gehen und damit in Konflikt mit den Interessen des Naturschutzes treten. Eine ähnliche Entwicklung findet in Südamerika statt. In Brasilien soll der Sojaanbau von knapp 23 Mio. ha im Jahr 2005 (nach FAODaten) auf ca. 100 Mio. ha ausgedehnt werden (Kaltner et al. 2005; Flaskerud 2003). Diese Erweiterung der landwirtschaftlichen Flächennutzung würde nach Flaskerud (2003) im Mittel zu ca. 42 Prozent auf Cerradoflächen, zu 7 Prozent auf Amazonasregenwald und zu 51 Prozent auf Weideland entfallen.

Generell kann die Substitution von fossilen durch nachwachsende Rohstoffe zur Verminderung von Treibhausgasemissionen beitragen, wobei sich je nach Kulturpflanze, Anbauverfahren und Landnutzungsänderungen allerdings erhebliche Unterschiede bis hin zu Mehrbelastungen ergeben können (OECD, ITF 2008). Zudem bestehen große Unsicher- heiten in der Gesamtbewertung, da wesentliche Einflussfaktoren wie z. B. die Lachgas $\left(\mathrm{N}_{2} \mathrm{O}\right)$-Emissionen noch nicht hinreichend in die Ökobilanzierungen einbezogen wurden (Crutzen et al. 2007). Die Klimawirkung von Biodiesel aus Sojaöl und Palmöl fällt unter Anrechnung der Landnutzungsänderungen insbesondere durch die hohen Flächenerfordernisse bei Soja und die Trockenlegung von Torfböden bei Palmöl - gegenüber herkömmlichem Diesel insgesamt deutlich negativ aus (Wuppertal Institut et al. 2008). Fargione et al. (2008) berechneten die ,biofuel carbon debt“, d. h. die Menge an Kohlenstoff, die infolge der Konversion natürlicher Ökosysteme für den Anbau von Energiepflanzen durch Verbrennung oberirdischer Biomasse oder mikrobiellen Humusabbau in den folgenden fünfzig Jahren freigesetzt wird. Die Zeitspanne, die rechnerisch erforderlich wäre, um diese zusätzlichen Emissionen durch die fortgesetzte Nutzung von Biokraftstoffen wieder einzusparen (quasi die klimarelevante Amortisationszeit), liegt zwischen 17 Jahren bei Bioethanol aus Zuckerrohr im Cerrado und 420 Jahren bei Biodiesel aus Palmöl auf TorfRegenwaldböden mit hohem Kohlenstoffgehalt in Indonesien und Malaysia. In Indonesien entfällt ein Viertel der Regenwaldflächen auf Torfböden. Bis 2030 kann mit 50-ProzentAnteil Torfböden und einer entsprechend hohen ,biofuel carbon debt“" gerechnet werden (Hooijer et al. 2006).

Für Deutschland ermittelten Wuppertal Institut et al. (2008) anhand der BAU-Szenarien (siehe oben) die durch Flächennutzungsänderungen induzierten Treibhausgasemissionen. Dabei wurde angenommen, dass der Mehrbedarf an Biodiesel bis 2030 auf der Basis von Palmöl und Sojaöl gedeckt wird. Die Ergebnisse zeigen, dass der voraussichtliche Verbrauch von Biodiesel im Jahr 2030 deutlich mehr Treibhausgase (THG) durch Inanspruchnahme von Produktionsflächen im Ausland freisetzen würde, als insgesamt durch den Einsatz von Biodiesel eingespart werden könnte (23 bis 37 Mio. t). Werden dagegen die THG-Emissionen berechnet, die sich aus dem Netto-Flächen-Mehrbedarf durch den gesamten Verbrauch aller landwirtschaftlich basierter Güter ergeben ${ }^{3}$, dann würde sich der NettoEffekt durch den Einsatz von Biodiesel in 
2030 auf 1 Mio. t THG-Minderung verringern bzw. würde zu 10 Mio. t THG-Mehremissionen (BAU I bis II) führen. Diese Ergebnisse zeigen, dass es bei Berücksichtigung der Trends vor dem Zeitraum 2040 bis 2050 nicht zu einer Netto-Klimaentlastung durch die von Deutschland importierten Biodieselmengen kommen würde.

Dies ist darauf zurückzuführen, dass THG-Emissionen aus indirekten Landnutzungsänderungen selbst dann zu erwarten sind, wenn die Maßgaben der vorgesehenen Biomasse-Nachhaltigkeitsverordnung erfüllt würden, wonach die Importe nicht von Umwandlungsflächen stammen dürfen. ${ }^{4}$ Denn die gestiegene Netto-Flächen-Inanspruchnahme hätte zur Folge, dass zwangsläufig an anderer Stelle eine Ausweitung der Anbaufläche induziert würde, da durch indirekte Verdrängungseffekte $u$. a. der Anbau von Nahrungsmitteln zur Versorgung der einheimischen Bevölkerung auf neue Flächen ausweichen müsste, deren Rodung zu Lasten natürlicher Ökosysteme ginge.

Nach Untersuchungen des „Stockholm International Water Institute" kann die Ausdehnung des Energiepflanzenanbaus neben den dargestellten Auswirkungen auf den Naturhaushalt und das Klima auch zu einem signifikanten Druck auf die globalen Wasserressourcen beitragen. In den kommenden Jahrzehnten würde der Wasserbedarf für Bioenergie danach wesentlich zum Gesamtwasserbedarf weltweit beitragen - vor allem in Lateinamerika und in Afrika südlich der Sahara. Daher sollten die Folgewirkungen hoher Bioenergieproduktion und ihres Wasserbedarfs in den betroffenen Regionen untersucht werden (Lundqvist et al. 2008).

\section{Elemente alternativer Entwicklungspfade}

In Anbetracht der dargestellten Problematik ist die Suche nach alternativen Entwicklungspfaden erforderlich, die den landwirtschaftlichen Flächenbedarf verringern und damit Nutzungskonkurrenzen mindern sowie positive Beiträge zum Klimaschutz erbringen. Eine versorgungsseitig vorrangige Strategie liegt in

a) der primär stofflichen Verwendung von Non-Food-Biomasse, b) ihrer kaskadenförmigen Nutzung und

c) der energetischen Verwertung organischer Rest- und Abfallstoffe.

Beim Einsatz von Non-Food-Biomasse für die stoffliche Nutzung fällt die mögliche Umweltentlastung generell höher aus als wenn diese für die Erzeugung von Bioenergie verwendet wird (Weiß et al. 2003, 2004), wobei der Einsatz in stationären Anlagen zur Kraftwärmeerzeugung effizienter ist als jener für Biokraftstoffe. Wird die Biomasse dagegen zuerst stofflich eingesetzt, möglichst mehrfach rezykliert und erst danach energetisch verwertet (Kaskadenprinzip), so wird potenziell eine wesentlich höhere Umweltentlastung erzielt als bei der primär energetischen Verwendung. Werden Biokraftstoffe aus lignocellulosehaltigen Restund Abfallstoffen hergestellt (die sog. 2. Generation), ergeben sich deutliche Umweltentlastungen im Vergleich zu Biokraftstoffen der ersten Generation wie beispielsweise solchen aus Rapsöl (Zah et al. 2007). Die Verwendung von Rest- und Abfallstoffen hat zudem den Vorteil, keine zusätzlichen Flächen zu benötigen. Allerdings wird hier zu klären sein, wie sich die Konkurrenz der Nachfrage nach Material zwischen etablierten Industrien (z. B. Holzwerkstoff-, Papier- und Pappeindustrie), der Verwendung für Wärme/Strom (z. B. Pelletheizungen mit oder ohne Kraftwärmekopplung) und dem Einsatz in Biomass-to-LiquidAnlagen auswirken wird.

Weitere alternative Entwicklungspfade zur Reduzierung der Nutzungskonflikte sind:

1. Ersatz von Biokraftstoffen durch Biogas für Strom und Wärme beim inländischen Anbau;

2. Verminderung der Tierproduktion im Inland;

3. Ersatz der Stromerzeugung aus Anbaubiomasse durch Photovoltaik;

4. Verringerung des Treibstoffverbrauchs der Fahrzeugflotte;

5. Verminderung des Verbrauchs tierisch basierter Ernährung;

6. Verminderung des Anteils nicht verwerteter Lebensmittel in Handel, Gewerbe und Haushalten.

Die jeweiligen Potenziale dieser alternativen Elemente für eine nachhaltigere globale landwirtschaftliche Flächennutzung wurden exemplarisch am Beispiel von Deutschland eingeschätzt. Es wurde deutlich, dass Strategien auf 
Tab. 1: Auswirkungen angebots- und verbrauchsseitiger Strategien zur Verringerung des globalen Flächenbedarfs und der deutschen Treibhausgasemissionen*

\begin{tabular}{|c|c|c|}
\hline Angebotsseitig & $\begin{array}{l}\text { Einsparpotenzial globale } \\
\text { Flächenbelegung (2030) }\end{array}$ & $\begin{array}{l}\text { Einsparpotenzial THG- } \\
\text { Äquivalente }\end{array}$ \\
\hline \multirow{2}{*}{ Biogas statt Biokraftstoffe } & konstant & 15,8 - 17,9 Mio. $\mathrm{t}$ \\
\hline & $100 \mathrm{~m}^{2}$ pro Kopf & n. a. \\
\hline Photovoltaik statt Biogas & $100 \mathrm{~m}^{2}$ pro Kopf & 2,7-3,0 Mio. $t$ \\
\hline Verminderung inländischer Tierproduktion & +/- bei gleichem Konsum & +/- bei gleichem Konsum \\
\hline \multicolumn{3}{|l|}{ Verbrauchsseitig } \\
\hline $\begin{array}{l}\text { Verminderung tierisch basierter Ernährung } \\
\text { nach Empfehlungen der DGE }\end{array}$ & 400 bis $500 \mathrm{~m}^{2}$ pro Kopf & n. a. (synerg.) \\
\hline $\begin{array}{l}\text { Verminderung der Verluste von Nah- } \\
\text { rungsmitteln in Haushalten }\end{array}$ & ca. $200 \mathrm{~m}^{2}$ pro Kopf & n. a. (synerg.) \\
\hline $\begin{array}{l}\text { Emissionsminderung bei der } \mathrm{PkW} \text { - } \\
\text { Fahrzeugflotte auf } 130 \mathrm{~g} \mathrm{CO}_{2} / \mathrm{km}\end{array}$ & 500 bis $600 \mathrm{~m}^{2}$ pro Kopf & $\begin{array}{l}\text { 29,6 Mio. } t \\
23 \% \text { Diesel, 27\% Benzin }\end{array}$ \\
\hline
\end{tabular}

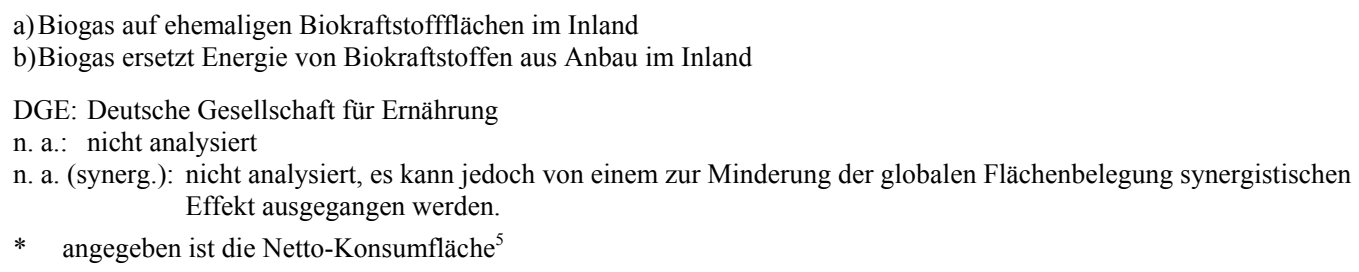

Quelle: Wuppertal Institut et al. 2008

der Angebotsseite wie die Optionen (1) bis (3) zwar einen Beitrag zur nationalen THGEmissionsminderung leisten können, bezogen auf die globale Flächennutzung aber keinen oder nur einen relativ geringen Effekt zeigen würden (siehe Tab. 1 nächste Seite). Eine Verminderung der inländischen Tierproduktion bei gleich bleibendem Konsum würde durch eine Steigerung der Importe von Fleisch- und Milchprodukten sowie Futtermitteln kompensiert werden, so dass sich an der Größenordnung der global belegten Fläche nichts ändern würde.

Eine Veränderung des Verbrauchs in Produktion und Konsum ist daher zum Erreichen einer global ausgewogenen Flächennutzung unumgänglich (Optionen 4 bis 6). Der globale Flächenverbrauch Deutschlands im Jahr 2030 könnte um etwa $500 \mathrm{~m}^{2}$ pro Kopf reduziert werden, wenn auf den Einsatz von Biokraftstoffen gänzlich verzichtet und statt dessen der
Treibstoffverbrauch der Pkw-Fahrzeugflotte (4) um ca. 30 Prozent (entsprechend der diskutierten Begrenzung des $\mathrm{CO}_{2}$-Ausstoßes auf $130 \mathrm{~g}$ $\mathrm{CO}_{2}$ pro $\mathrm{km}$ ) verringert würde. Der Klimaschutzeffekt dieser Maßnahme wäre mit ca. 29,6 Mio. t Einsparpotenzial an Treibhausgasen deutlich größer als der Effekt, der durch den Quoteneinsatz von Biokraftstoffen maximal erreicht werden könnte.

Auch eine Verminderung des Verbrauchs tierisch basierter Ernährung (5) auf ein Niveau, das von der Deutschen Gesellschaft für Ernährung empfohlen wird, würde einen NettoEinspareffekt von bis zu etwa $500 \mathrm{~m}^{2}$ pro Kopf erbringen. Zusätzlich könnte eine Verminderung des Anteils nicht verwerteter Lebensmittel in Haushalten (6) einen Einspareffekt von mindestens $200 \mathrm{~m}^{2}$ pro Kopf erbringen, wobei noch offen bleibt, welches zusätzliche Potenzi- 
al sich durch Verminderung der Verluste im Handel ergeben würde.

Insgesamt wäre ein Einsparpotenzial möglich, welches wesentlich zu einer nachhaltigeren globalen landwirtschaftlichen Flächennutzung Deutschlands beitragen würde und sogar einen gewissen Raum böte zur Produktion von Biomasse für die primär stoffliche Verwendung (z. B. für Biopolymere) und für die Nahrungsmittelversorgung zur Deckung des wachsenden weltweiten Bedarfs oder für die Verwendung größerer Flächen für ökologischen Landbau. Das Potenzial könnte de facto nur über Maßnahmen zur Veränderung des Verbrauchs genutzt werden, wobei diese sich synergistisch auf den Ressourcen- und Klimaschutz auswirken würden.

\section{Schlussfolgerungen und Empfehlungen}

Um eine nachhaltige Produktion und Verwendung nachwachsender Rohstoffe im nationalen und internationalen Kontext sicher zu stellen und dafür insbesondere die globalen Flächennutzungskonkurrenzen $\mathrm{zu}$ vermindern, erscheint die Überprüfung verschiedener Instrumente und der damit verfolgten Strategien und politischen Handlungsziele angezeigt (Wuppertal Institut et al. 2008; Bringezu und Schütz 2008). Hierzu gehören

- die Überprüfung der Förderung des Energiepflanzenanbaus: Diese müsste sich insbesondere mit der Vergabe der Energiepflanzenprämie auseinandersetzen, die im Zuge der anstehenden Überprüfung der Gemeinsamen Agrarpolitik der EU (,Health Check") gestrichen werden sollte.

- das Hinterfragen einer Extensivierung der Landwirtschaft: Dies meint erforschen, entwickeln und fördern von Anbauformen, die zum einen hoch produktiv und zum anderen umwelt- und landschaftsverträglich sind (z. B. Precision-Farming; NiedrigEnergie-Treibhäuser, Intensivanbau gekoppelt mit Ausgleichsflächen und Randstreifen etc.), aber auch die Intensivierung des Ökolandbaus.

- die Mobilisierung von Holzrohstoffen im Inland: Über sie wäre die stoffliche Verwendung von nachwachsenden Rohstoffen
(Nawaro) in Bereichen zu fördern, die nicht mit Nahrungsmitteln konkurrieren, und die Nutzung bislang nicht ausgeschöpfter Potenziale insbesondere im Kleinprivatwald anzustreben, bei der Mobilisierungshemmnisse über technologische, logistische und organisatorische Ansätze überwunden werden könnten.

- die Steuerung der Nawaro-Nachfrage: Hier wird empfohlen, die gesetzlich verbindlichen Biotreibstoffquoten nicht wie geplant zu erhöhen, sondern eher zurückzufahren oder zumindest auf dem derzeitigen Niveau einzufrieren, wie es u. a. auch der Sachverständigenrat für Umweltfragen (2007) empfohlen hat. Hinsichtlich des Nawaro-Bonus im EEG sind vor allem Entwicklungen zu bedenken, die bei Fortdauer der bestehenden Trends zu erheblichen Anteilen der Gesamtflächennutzung führen, wie dies z. B. bei Mais für die Biogaserzeugung zu erwarten ist.

- der Ausbau von Strategien für ein nachhaltiges Ressourcenmanagement: Unterstützung von Exportländern des Südens beim Aufbau eines nachhaltigen Ressourcenmanagements, das u. a. ein stabiles Verhältnis von Natur-, Forst- und Landwirtschaftsflächen auf jeweils nachhaltigem Niveau gewährleistet. Deutschland ist wie andere Länder der EU aufgefordert, ein nachhaltiges Ressourcenmanagement zu etablieren (gemäß der thematischen Strategie zur nachhaltigen Nutzung natürlicher Ressourcen). Dabei gilt es, biotische und abiotische Ressourcen ebenso wie die Flächennutzung integrativ zu betrachten. Hierzu wäre ein hinreichendes Monitoring zu etablieren, das die relevanten transregionalen und transnationalen Implikationen der regionalen bzw. nationalen Aktivitäten ausweist (wie die globale Flächennutzung durch inländischen Verbrauch agrarischer oder forstlicher Güter).

- die Weiterentwicklung politischer Instrumente: Dies hat insbesondere auf Maßnahmen zur Förderung der Material- und Energieeffizienz in Produktion und Konsum bei gleichzeitig absoluter Verminderung des Ressourcenverbrauchs zu zielen.

- das Einbinden internationaler Programme und Institutionen: Dazu gehört der Aufbau eines internationalen Satelliten gestützten Flächennutzungskatasters für ein integrier- 
tes Management verschiedener biotischer und abiotischer Ressourcen (Land-, Forstwirtschaft, Bergbau, Naturschutz) sowie die Einflussnahme auf UNEP und UNEnergy sowie in der Global Bioenergy Partnership (GBEP), um auch im Sinne des Vorsorgeprinzips eine internationale Übereinkunft darüber zu erzielen, dass (a) Biomasse generell primär stofflich für Ernährung oder Materialzwecke eingesetzt und (b) erst danach der Energiegehalt aus den organischen Abfällen genutzt werden sollte („Kaskadenprinzip“); der flächenmäßige Anbau von Energiepflanzen sollte die Ausnahme bleiben.

Eine längerfristige Aufgabe besteht im schrittweisen Aufbau eines internationalen nachhaltigen Ressourcenmanagements, bei dem eine nachhaltige Biomassestrategie eingebettet wäre in ein internationales Abkommen zur nachhaltigen Nutzung biotischer und abiotischer Rohstoffe und zu einer nachhaltigen Flächennutzung (Bleischwitz, Bringezu 2007). Zu diesem Zweck sollten bi- und multilaterale Vereinbarungen ausgebaut werden, um Ressourcen-, Klima- und Naturschutz synergistisch umzusetzen und zugleich über eine gesteigerte Ressourcenproduktivität die technologisch-institutionelle und sozioökonomische Entwicklung der Partner zu fördern.

\section{Anmerkungen}

1) $\mathrm{Zu}$ den Business-as-usual(BAU)-Szenarien siehe Kap. 4 in diesem Beitrag.

2) Den Zusammenhang zwischen Wachstum von Bioenergienutzung und Weltmarktpreisen für Nahrungs- und Bioenergie-Feldfrüchte hat das International Food Policy Research Institute (IFPRI) mithilfe des „International Model for Policy Analysis of Agricultural Commodities and Trade“ (IMPACT) untersucht (Rosegrant et al. 2006).

3) Bei dieser Berechnung wird berücksichtigt, dass der für die Ernährung erforderliche Flächenbedarf in Folge von Ertragssteigerungen sinken wird und die zunehmende Nachfrage nach nachwachsenden Rohstoffen teilweise kompensiert wird.

4) Hier wird auf den Entwurf einer Verordnung über Anforderungen an eine nachhaltige Erzeugung von zu Biokraftstoffen verwendeter
Biomasse Bezug genommen (Biomasse-Nachhaltigkeitsverordnung - BioNachV) (Stand 24.10.2007).

5) Mit Netto-Konsumfläche wird die landwirtschaftliche Anbaufläche bezeichnet, die tatsächlich erforderlich ist, um das Produkt aus Nawaro mit der entsprechenden Menge zu erhalten. Zum Beispiel wird dabei die Anbaufläche von Raps nur mit dem Anteil der Erzeugung von Biodiesel zugerechnet, der sich nach Aufteilung der Produktmengen für die Kraftstoff- bzw. Futtermittelproduktion ergibt; ein weiterer Anteil der gesamten Anbaufläche würde dann auf die Tierproduktion zugerechnet (z. B. 40 Prozent Öl aus Rapssaat zur Biodieselherstellung und 60 Prozent Ölpresskuchen aus Rapssaat für die Tierfütterung). Dagegen wird mit Brutto-Produktionsfläche die landwirtschaftliche Anbaufläche bezeichnet, die real erforderlich ist, um den Nawaro mit der entsprechenden Menge unabhängig von der weiteren Nutzung bereit zu stellen. Zum Beispiel wird dabei die Anbaufläche von Raps vollständig der Erzeugung von Biodiesel zugerechnet, wie dies z. B. in den Datensätzen der Fachagentur nachwachsende Rohstoffe der Fall ist (FNR 2006).

\section{Literatur}

Biofuelwatch; Carbon Trade Watch/TNI; Corporate Europe Observatory et al., 2007: Agrofuels - Towards a reality check in nine key areas. http://www.corporateeurope.org/docs/AgrofuelsRea lityCheck.pdf (download 8.8.08)

Bleischwitz, R.; Bringezu, S., 2007: Globales Ressourcenmanagement: Konfliktpotenziale und Grundzüge eines Global Governance-Systems. SEF Policy Paper 27, Stiftung Entwicklung und Frieden, Bonn; http://www.sef-bonn.org/download/publikationen/ policy_paper/pp_27_de.pdf (download 8.9.08)

Bringezu, S.; Schütz, H., 2008: Ausbau der Energiepflanzennutzung und Flächenkonkurrenz national und international. Gutachten für den Deutschen Bundestag - vorgelegt dem Büro für Technikfolgen-Abschätzung beim Deutschen Bundestag (TAB) - vom Wuppertal Institut für Klima, Umwelt, Energie GmbH, Wuppertal 2.6.2008

Busch, R., 2008: Untersuchung zur nachhaltigen Nutzung nachwachsender Rohstoffe: Globale Flächenbelegung Deutschlands für Produktion und Konsum tierischer Nahrungsmittel. Diplomarbeit an der Humboldt-Universität zu Berlin, Geographisches Institut, sowie persönliche Mitteilungen 
Colchester, M.; Jiwan, N.; Andiko; S. et al., 2006: Promised Land: Palm Oil and Land Acquisition in Indonesia - Implications for Local Communities and Indigenous Peoples. Forest Peoples Programme, Perkumpulan Sawit Watch, HuMA and the World Agroforestry Centre (Hg.); http://www.forestpeoples. org/documents/prv_sector/oil_palm/promised_land_ eng.pdf (download 10.9.08)

Crutzen, P.J.; Mosier, A.R.; Smith, K.A. et al., 2007: $\mathrm{N}_{2} \mathrm{O}$ release from agro-biofuel production negates global warming reduction by replacing fossil fuels. Atmospheric Chemistry and Physics Discussions 7 (2007), S. 11191-11205

EC - European Commission, 2006: Biofuels Progress Report. Report on the progress made in the use of biofuels and other renewable fuels in the Member States of the European Union. Commission Staff working document, SEC(2006) 1721/2, Brussels, Belgium

FAO - Food and Agriculture Organization, 2003: World agriculture: towards 2015/2030. An FAO perspective. Rome, London

FAO - Food and Agriculture Organization, 2006: World agriculture: towards 2030/2050. Interim report, Rome

Fargione, J.; Hill, J.; Tilman, D. et al., 2008: Land Clearing and the Biofuel Carbon Debt. Science 319/5867 (2008), S. 1235-38

Flaskerud, G., 2003: Brazil's Soybean Production and Impact. NDSU Extension Services, North Dakota State University of Agriculture and Applied Science, and U.S. Department of Agriculture; http:// www.ag.ndsu.edu/pubs/agecon/market/eb79.pdf (download 8.9.08)

FNR - Fachagentur Nachwachsende Rohstoffe, 2006: Biokraftstoffe - eine vergleichende Analyse. Gülzow

Gömann, H.; Kreins, P.; Breuer, T. et al., 2007: Nutzungskonkurrenzen durch die Förderung von Biogas und anderen Energieträgern. In: Agrarspektrum, Bd. 40, Energie aus Biomasse - weltwirtschaftliche, ressourcenökonomische und produktionstechnische Perspektiven. S. 135-150

Grieg-Gran, M.; Haase, M.; Kessler, J.J. et al., 2007: The Dutch economic contribution to worldwide deforestation and forest degradation. Report prepared for Greenpeace Netherlands. London, Amsterdam

Hazell, P.; Wood, S., 2008: Drivers of change in global agriculture. Phil. Trans. R. Soc. B 363 (2008), S. 495-515

Hooijer, A.; Silvius, M.; Wösten, H. et al., 2006: Peat- $\mathrm{CO}_{2}$. Assessment of $\mathrm{CO}_{2}$ emissions from drained peatlands in SE Asia. Delft Hydraulics Report Q3943. Amsterdam

Kaltner, F.; Azevedo, G.F.P.; Campos, I.A. et al., 2005: Liquid Biofuels for Transportation in Brazil. Potential and Implications for Sustainable Agriculture and Energy in the $21^{\text {st }}$ Century. Submitted Report $\left(2^{\text {nd }}\right.$ Draft); http://www.fbds.org.br/IMG/pdf/doc -116.pdf (download 8.9.08)

Lundqvist, J.; de Fraiture, C.; Molden, D. et al., 2008: Saving Water: From Field to Fork. Curbing losses and wastage in the food chain. Draft for CSD, May 2008. Stockholm; http://www.siwi.org/ documents/Resources/Papers/Paper_13_Field_to_ Fork.pdf (download 8.9.08)

Meó Consulting Team; Faserinstitut Bremen; novaInstitut GmbH, 2007: Marktanalyse Nachwachsende Rohstoffe Teil II. FNR (Hg.), Gülzow

Nusser, M.; Sheridan, P.; Walz, R. et al., 2007: Makroökonomische Effekte des Anbaus und der Nutzung von nachwachsenden Rohstoffen. Studie für das Bundesministerium für Ernährung, Landwirtschaft und Verbraucherschutz vertreten durch die Fachagentur Nachwachsende Rohstoffe e.V., Gülzow; http://www.isi.fhg.de/t/projekte/Makro-NAWAROEndbericht.pdf (download 8.9.08)

OECD - Organisation for Economic Co-operation and Development, 2008: OECD Environmental Outlook to 2030. OECD, Paris

OECD-FAO - Organisation for Economic Cooperation and Development; Food and Agriculture Organization, 2007: Agricultural Outlook: 20072016. OECD, Paris

OECD-ITF - Organisation for Economic Cooperation and Development; International Transport Forum, 2008: Biofuels: Linking Support to Performance. Round Table 138, Paris

Rosegrant, M.W.; Msangi, S.; Sulser, T. et al., 2006: Biofuels and the global food balance. In: Hazell, P.; Pachauri, R.K. (Hg.): Bioenergy and Agriculture Promises and Challenges. http://www.ifpri.org/2020/ focus/focus14/focus14.pdf (download 8.9.08)

Searchinger, T.; Heimlich, R.; Houghton, R.A. et al., 2008: Use of U.S. croplands for biofuels increases greenhouse gases through emissions from land use changes. In: Science 319/5867 (2008), S. 1238-40

SRU - Sachverständigenrat für Umweltfragen, 2007: Klimaschutz durch Biomasse. Sondergutachten - Hausdruck. Berlin

UN-Energy, 2007: Sustainable Bioenergy: A Framework for Decision Makers. ftp://ftp.fao.org/docrep/ fao/010/a1094e/a1094e00.pdf (download 8.9.08) 
Weiß, M.; Bringezu, S.; Heilmeier, H., 2003/2004: Energie, Kraftstoffe und Gebrauchsgüter aus Biomasse: Ein flächenbezogener Vergleich von Umweltbelastungen durch Produkte aus nachwachsenden und fossilen Rohstoffen. Zeitschrift für angewandte Umweltforschung (ZAU) 15-16/3-5 (2003/2004), S. 361-378

Wuppertal Institut für Klima, Umwelt, Energie; Fraunhofer-Institut für Umwelt-, Sicherheits- und Energietechnik (UMSICHT); Institut für Energieund Umweltforschung (IFEU) Heidelberg, 2008: Optionen einer nachhaltigen Flächennutzung und Ressourcenschutzstrategien unter besonderer Berücksichtigung der nachhaltigen Versorgung mit nachwachsenden Rohstoffen. Vorhaben Z 6-91 054/82, Forschungskennzahl (FKZ) 20593153 , Endbericht Mai 2008. Wuppertal

Wuppertal Institut; RWI, 2008: Nutzungskonkurrenzen bei Biomasse - Auswirkungen der verstärkten Nutzung von Biomasse im Energiebereich auf die stoffliche Nutzung in der Biomasse verarbeitenden Industrie und deren Wettbewerbsfähigkeit durch staatlich induzierte Förderprogramme. Ein Studie des Wuppertal Instituts für Klima, Umwelt, Energie GmbH (WI) und des Rheinisch-Westfälischen Institut für Wirtschaftsforschung (RWI) für das Bundesministerium für Wirtschaft und Technologie (BMWi). Endbericht 25. April 2008. Wuppertal, Essen

Zah, R.; Böni, H.; Gauch, M. et al., 2007: Ökobilanz von Energieprodukten: Ökologische Bewertung von Biotreibstoffen. Schlussbericht. EMPA, St. Gallen

\section{Kontakt}

Dr. Stefan Bringezu

Wuppertal Institut für Klima, Umwelt, Energie

$\mathrm{GmbH}$

Döppersberg 19, 42103 Wuppertal

E-Mail: stefan.bringezu@wupperinst.org

$《 \gg$

\section{Auswirkungen der Konkurrenz zwischen Nahrungsmittel- und Bioenergieproduktion auf Landwirtschaft, Gesellschaft und Umwelt}

\author{
von A. Heißenhuber, M. Demmeler, S. Rauh, \\ TU München-Weihenstephan
}

In den Jahren 2003 bis 2007 gelang es, den Anbau von Energiebiomasse zu forcieren. Das Fruchtfolgespektrum in Deutschland wurde dementsprechend in Richtung sog. Energiekulturen verschoben. Aufgrund aktueller Entwicklungen auf den Agrarund Energiemärkten kommt es nun verstärkt zur Konkurrenz zwischen der Nahrungsmittel- und der Energieproduktion um den knappen Faktor Boden. Die Bioenergielinien sind aber mittlerweile in ihrer Wettbewerbskraft der „klassischen“ Landwirtschaft unterlegen. Aus gesellschaftlicher Sicht ist die Herstellung von Bioenergie aus Reststoffen zu bevorzugen, bei der es zu keiner Verdrängung etablierter Produktionsverfahren kommt. Auf jeden Fall gilt es zu prüfen, in wieweit jeglicher Biomasseanbau einer Naturverträglichkeitsprüfung standhält. Unter Umständen führt eine regionsspezifische Untersuchung zu Einschränkungen im Anbauumfang bestimmter Kulturen im Sinne des Ressourcenschutzes.

\section{Ausgangssituation}

Die Produktion nachwachsender Rohstoffe erschien für die Landwirtschaft bis vor zwei Jahren noch als gute Möglichkeit, eine zusätzliche Einkommensquelle zu erschließen. Maßgeblich dafür waren z. B. die staatlich festgelegten Einspeisetarife für Strom aus Biogasanlagen. Die Absatzmöglichkeiten für Getreide und Raps an die Hersteller von Biosprit versprachen ebenfalls einen höheren Erlös als auf den Nahrungsmärkten bzw. zumindest eine Verringerung des Preisdruckes. Die Folge dieser Entwicklung war ein starker Ausbau der Anbaufläche für nachwachsende Rohstoffe. Zwischen 2003 und 2007 hat sie sich mehr als verdoppelt auf heute ca. zwei Millionen Hektar 